University of Nebraska - Lincoln

DigitalCommons@University of Nebraska - Lincoln

Other Publications in Zoonotics and Wildlife

Disease

Wildlife Disease and Zoonotics

2014

\title{
Sources of bovine tuberculosis in the United States
}

Kimberly Tsao

Colorado State University, kim.tsao@colostate.edu

Suelee Robbe-Austerman

USDA APHIS Veterinary Services

Ryan S. Miller

Colorado State University

Katie Portacci

USDA APHIS Veterinary Services

Daniel A. Grear

USDA APHIS Veterinary Services, dgrear@usgs.gov

See next page for additional authors

Follow this and additional works at: https://digitalcommons.unl.edu/zoonoticspub

Tsao, Kimberly; Robbe-Austerman, Suelee; Miller, Ryan S.; Portacci, Katie; Grear, Daniel A.; and Webb, Colleen T., "Sources of bovine tuberculosis in the United States" (2014). Other Publications in Zoonotics and Wildlife Disease. 185.

https://digitalcommons.unl.edu/zoonoticspub/185

This Article is brought to you for free and open access by the Wildlife Disease and Zoonotics at DigitalCommons@University of Nebraska - Lincoln. It has been accepted for inclusion in Other Publications in Zoonotics and Wildlife Disease by an authorized administrator of DigitalCommons@University of Nebraska Lincoln. 
Authors

Kimberly Tsao, Suelee Robbe-Austerman, Ryan S. Miller, Katie Portacci, Daniel A. Grear, and Colleen T. Webb 


\title{
Sources of bovine tuberculosis in the United States
}

\author{
Kimberly Tsao ${ }^{a, *}$, Suelee Robbe-Austerman ${ }^{b}$, Ryan S. Miller ${ }^{a, c}$, Katie Portacci ${ }^{c}$, Daniel A. Grear ${ }^{c}$, \\ Colleen Webb ${ }^{a}$ \\ ${ }^{a}$ Department of Biology, Colorado State University, Fort Collins, CO 80523, USA \\ ${ }^{\mathrm{b}}$ USDA APHIS Veterinary Services, Science Technology and Analysis Services (STAS), National Veterinary Services Laboratories, Ames, IA, USA \\ ${ }^{\text {c } U S D A}$ APHIS Veterinary Services, STAS, Center for Epidemiology and Animal Health, Fort Collins, CO, USA
}

\section{A R T I C L E I N F O}

\section{Article history:}

Received 25 July 2014

Received in revised form 17 September 2014

Accepted 18 September 2014

Available online 26 September 2014

\section{Keywords:}

Mycobacterium bovis

Clonal complex

Spillover

Reservoir

\begin{abstract}
A B S T R A C T
Despite control and eradication efforts, bovine tuberculosis continues to be identified at low levels among cattle in the United States. We evaluated possible external sources of infection by characterizing the genetic relatedness of bovine tuberculosis from a national database of reported infections, comparing strains circulating among US cattle with those of imported cattle, and farmed and wild cervids.

Farmed cervids maintained a genetically distinct Mycobacterium bovis strain, and cattle occasionally became infected with this strain. In contrast, wild cervids acted as an epidemiologically distinct group, instead hosting many of the same strains found in cattle, and the data did not show a clear transmission direction. Cattle from Mexico hosted a higher overall richness of strains than US cattle, and many of those strains were found in both US and Mexican cattle. However, these two populations appeared to be wellmixed with respect to their $M$. bovis lineages, and higher resolution data is necessary to infer the direction of recent transmission.

Overall patterns of both host and geographic distributions were highly variable among strains, suggesting that different sources or transmission mechanisms are contributing to maintaining different strains.
\end{abstract} (c) 2014 Elsevier B.V. All rights reserved.

\section{Introduction}

\subsection{Epidemiology and evolution}

Mycobacterium bovis, the causative agent of bovine tuberculosis (bTB), continues to infect cattle in the United States at a low level despite control and eradication efforts. Routes of transmission include shared feed, other environmental fomites, and direct contact. There are multiple possible recent infection sources of $M$. bovis transmission to US cattle, including: (1) infected cervids (deer and their relatives), and (2) cattle imported from bTB-endemic areas outside the US. Here we consider evidence for these potential sources in the context of $M$. bovis genetic relatedness.

Our inferences rely on relationships among strains, which we evaluate by reconstructing clonal complexes. The evolutionary model of clonal expansion that gives rise to these complexes is well supported in previous $M$. bovis population genetic studies (Gutiérrez Reyes et al., 2012; Smith, 2012; Smith et al., 2003, 2006). In this model, a founder strain spreads among many individuals, and as it reproduces and is transmitted, subsequent

\footnotetext{
* Corresponding author. Tel.: +1 5104954266 .

E-mail address: kim.tsao@colostate.edu (K. Tsao).
}

mutations produce a group of closely related strains, together forming a clonal complex. The low overall rates of horizontal transfer and mutation in $M$. bovis make these complexes easily identifiable, with many strains only varying at a single locus among genetic markers. Strains within the same clonal complex are inferred to have descended from a common founder strain. By identifying hosts (Collins et al., 1988) or geographic regions (Allen et al., 2013) from which members of a clonal complex are reported, we can make epidemiological inferences about the sources of these strains, based on the hosts and locations from which the other members of the complex are reported.

\subsection{Possible M. bovis sources}

Whether acting as reservoirs maintaining a pathogen or as incidental hosts only occasionally becoming infected, cervids can facilitate $M$. bovis transmission via direct or indirect contact with domestic cattle.

\subsubsection{Wild cervids}

Outside the US, wildlife reservoirs have been recognized to independently maintain and transmit $M$. bovis to cattle, impeding eradication in cattle populations. These wildlife reservoirs include 
European badger (Meles meles) in Europe (Woodroffe et al., 2005), brushtail possum (Trichosurus vulpecula) in New Zealand (Collins et al., 1988), Cape buffalo (Syncerus caffer) and greater kudu (Tragelaphus strepsiceros) in southern Africa (Bengis et al., 1996), elk (Cervus canadensis) and American bison (Bison bison) in Canada (Wobeser, 2009), and wild boar (Sus scrofa) in Europe (Naranjo et al., 2008).

In North America, $M$. bovis has been identified in populations of White-tailed deer (Odocoileus virginianus) (Miller and Sweeney, 2013; Smith, 1968), but published data on matching deer bTB genotypes to local cattle are not as extensive as in other bTB wildlife reservoir systems (Biek et al., 2012). Infection in both wild cervids and cattle in these areas have led to trade restrictions and altered wildlife management practices (O'Brien et al., 2006).

\subsubsection{Farmed cervids}

Farmed cervids are in some aspects managed similarly to cattle, including being fed from shared containers and being transported among farms. Their exposure risks and contact patterns are likely more similar to cattle than to their wild counterparts, so as an epidemiological host group we expect farmed cervids to contribute differently to cattle infection than do wild cervids.

\subsubsection{International imports}

Cattle imported from $M$. bovis-endemic countries could be periodically introducing the pathogen to US cattle. This would not be a new phenomenon, as pathogen introduction has a long global history as an unintended consequence of live imports. Countries that historically traded with the British Isles, including the US, Canada, New Zealand, Australia, and South Africa, still have M. bovis strains in the same clonal complex as strains currently present in the UK (Smith et al., 2011). However, here we are interested in international imports as a source of recent pathogen introductions (within the past two decades), leading to established infections in US cattle populations. Currently, only Canada, Mexico, and Australia are permitted to send live cattle to the United States.

\subsection{Evaluating potential external sources}

Genetic analyses of $M$. bovis from multiple host species have not previously been evaluated at this large of a spatiotemporal scale for the US. Here we summarize $M$. bovis genetic relatedness, geographic distribution, and host types for the most frequently detected strains in the US, or those causing the highest population-level disease burden. Based on these characteristics, wild cervids, farmed cervids, and imported cattle were all evaluated as possible source populations infecting US cattle.

\section{Materials and methods}

Data were provided by the National Veterinary Services Laboratories (NVSL) from a collection database of $M$. bovis isolates. These samples are a subset of reported cases in the US between 1989 and 2013. Prior to 2001, isolates were archived at NVSL sporadically with no standardized protocol. Approximately 40\% of bTB affected herds between 1989 and 2000 have at least one representative isolate in the database. After 2001 with formalized archiving procedures in place, $100 \%$ of US-origin affected herds, and $95 \%$ of imported cattle isolates were genotyped and included. The dataset also contained information about individual hosts, including species, production type (i.e., wildlife, game farm), year isolated, last state of residence, and country of origin (Appendix A). Of the 897 M. bovis records from cattle, 595 were from US cattle, 202 were from cattle imported from Mexico, 4 were from cattle imported from Canada, and 96 were of unknown origin. We included the strains from Canadian cattle in examining individual strain distributions, but focused on the larger sample from Mexico for further analyses of international imports. We assumed that strains in imported cattle were acquired in the cattle's country of origin, where bTB is endemic (USDA: APHIS, 2013), although the case reports were in the US. All 170 cervid samples, both farmed and wild, were from the US. Eighty-two reports came from other wildlife species (opossums, raccoons, coyotes, and feral pigs); these were not included in the analysis. One hundred ten samples had incomplete genetic data and were excluded from analysis, for a total of 1111 records.

Samples were identified based on spoligotyping, a categorization method commonly used in the Mycobacterium tuberculosis complex (reference database at http://www.mbovis.org (Smith and Upton, 2012)), which includes M. bovis. Spoligotypes identify groups of closely related strains based on "presence or absence... of spacer units in the chromosome" (Smith and Upton, 2012). Spoligotypes were grouped into families if they differed from at least one other member by no more than a single spacer deletion (Reyes et al., 2008). This case definition was based on the relative frequency of a single spacer deletion event (ca. 3 times higher) compared to multiple spacer deletion events. Additionally, eleven Variable Number of Tandem Repeat (VNTR) loci, 0424, 0577, 1644 (MIRU16), 1955, 2165 (ETRA), 2401, 2461, 2687 (MIRU24), 2996 (MIRU26), 3192 (MIRU31), 4052 (QUB-26) (Martinez et al., 2008) were characterized and used to further define structure within spoligotype families. Each unique combination of spoligotype and VNTR profile was defined as a strain.

We estimated strain richness among host groups by generating rarefaction curves. This procedure subsamples within a group to estimate rates at which new strains are detected, allowing us to account for different group sample sizes (numbers of reports). We used the "rarecurve" species accumulation curve function in the "vegan" package (Oksanen et al., 2012) in the R programming environment (Oksanen et al., 2012; R Core Team, 2012), to estimate strain detection rates in the host groups: US cattle, cattle imported from Mexico (henceforth "Mexican cattle"), farmed cervids, and wild cervids.

To visualize genetic relatedness among $M$. bovis strains, strains within each spoligotype family were aggregated into clonal complexes using eBURST (http://eburst.mlst.net) (Feil et al., 2004). Relationships among spoligotype families were not evaluated here, but have been described previously (Smith, 2012). Because they were grouped by spoligotype family, strains within the same clonal complex often, but not always, share the same spoligotypes. Relationships within clonal complexes are thus largely defined by VNTR profile similarity, with spoligotype treated as a single locus. Membership within a clonal complex was defined as sharing 11 of 12 loci (11 VNTR plus one spoligotype) with at least one other strain in the complex. We compared strains with respect to host group, production type, and country of origin. We included isolates from cattle of unknown origin to determine the most frequently reported strains, then patterns in those strain distributions were determined based on reports from known locations.

\section{Results}

\subsection{Strain richness}

We identified a total of 138 unique strains in 27 spoligotype families. Most spoligotype families were comprised of three to four clonal complexes, and numerous pairs and singleton strains.

The rarefaction curves show large differences in strain richness among US cattle, Mexican cattle, and cervids (Fig. 1). At a sample size of 60 reports from each group, Mexican cattle on average yield 


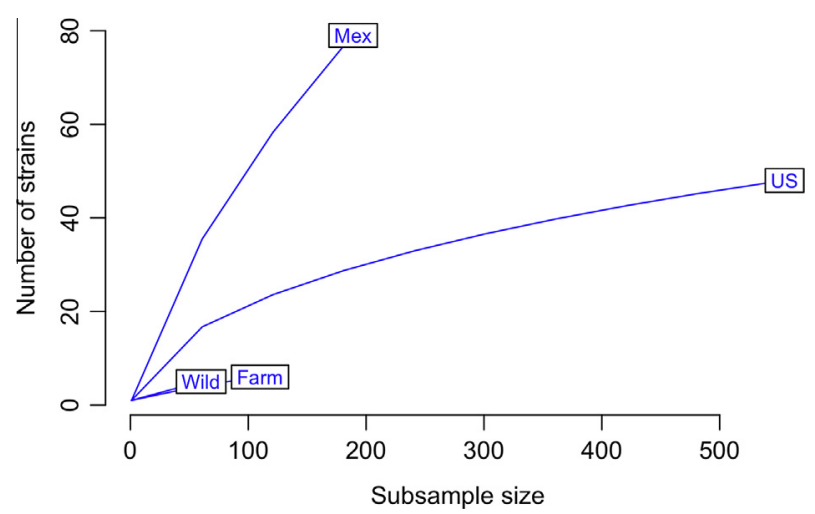

Fig. 1. Average counts of unique strains present in reports from Mexican cattle (Mex), US cattle (US), wild cervids (Wild) and farmed cervids (Farm), at increasing subsample sizes. Curves extend out to the actual sample size for each host group.

35.1 strains (standard error (SE) 2.9), US cattle 16.6 strains (SE 2.3), wild cervids 5 strains (SE 0, due to having the smallest sample size of 60), and farmed cervids 4.2 strains (SE 1.0). The rate of detecting new strains from Mexican cattle continues to rise steeply, suggesting much more diversity exists than is represented in this dataset, while the number of strains among US cattle approaches an asymptote around 40 strains. Both wild and farmed cervids have comparably low rates of detection of new strains. Restricting this analysis to index cases (excluding reports from multiple individuals in the same herds) yielded identically ranked and qualitatively similar results. The numbers of strains unique to, and shared among, host groups are summarized in Fig. 2.

\subsection{Variation in strain distribution patterns}

Individual strains vary widely in their distribution patterns with regard to host and geography. The five most frequently represented strains in the database (summing to 656 reports, or 59\%) each have unique distribution patterns (Fig. 3 and Appendix C).

The most frequently reported strain in the database had 195 reports ("strain A", spoligotype SB0145, strain ID 11 in Appendix A). Geographic distribution: reports of strain A were quite geographically limited (Fig. 3a); nearly all reports were from the state of Michigan fairly continuously since 1994 , with one report from Hawaii and one report from Mexican cattle in California. Host species distribution: strain A was reported from a range of host species (Fig. 4). In addition to cattle, there were also reports from wild and farmed deer and a farmed eland in Hawaii. Closely related strains: consistent with the clonal expansion model, strain A was reported from numerous individual hosts and is the estimated founder strain of its clonal complex (Fig. 3b). Most single locus

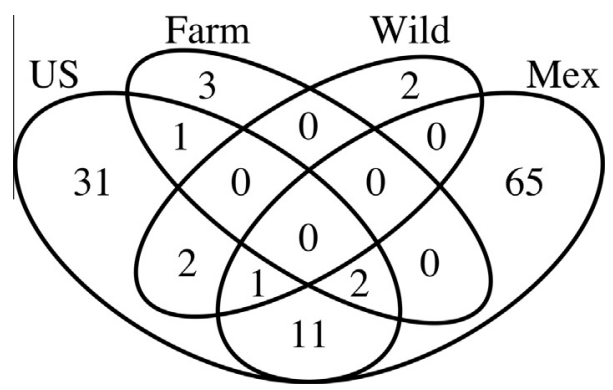

Fig. 2. Counts of unique strains reported by host type(s), shown as a Venn diagram. US: US cattle, Mex: Mexican cattle, Wild: wild cervids, Farm: farmed cervids. variants of this strain were also from Michigan (not shown in figure), but a single locus variant and double locus variant of strain A were also reported from Mexico.

The second most-frequently reported strain had 136 reports ("strain B", spoligotype SB0673, strain ID 2 in Appendix A). Geographic distribution: in contrast to strain A, strain B has a wide geographic range (Fig. 3c). In addition to appearing in multiple US states, it has also been reported from Mexican and Canadian cattle. Host species distribution: despite its wide geographic range, strain B was reported from a more limited range of host species; all reports but one (farmed elk from Michigan) were from cattle (Fig. 3d). Closely related strains: strain B is also the estimated founder of its clonal complex, giving rise to numerous variants (Fig. 3d). These variants are detected in US cattle, in Mexican cattle, and occasionally in both, making a single country of origin unclear. With the exception of three strains from wild deer and one from farmed cervids, strain B's variants were predominantly reported from cattle.

The third most frequently-reported strain had 129 reports ("strain C", spoligotype SB0265, strain ID 6 in Appendix A). Geographic distribution: while strain $C$ was reported over a fairly wide geographic range of 11 US states, it is notable that this strain has never been reported from imported cattle (Fig. 4), although other strains in the same spoligotype family were reported from Mexican cattle. Host species distribution: strain C was reported primarily from farmed cervids, and occasionally from cattle. There were no reports of strain C from wildlife in this dataset. Strain C comprises 101/110 (92\%) of reports from farmed cervids. Deer and elk classified as "production herd" animals were reported with strain C as early as 1991, and reports from farmed cervids continued for several years. Strain $C$ was reported less frequently from cattle, and in the later years of the dataset, with the earliest database record for cattle in 2001 (Appendix B). Closely related strains: strain $C$ was in a different spoligotype family, not closely related to strain A nor B. The other strains in the clonal complex with strain C consisted of a single locus variant reported from a farmed elk in 2009, and a strain with the same VNTR profile but different spoligotype (SB0121), reported from cattle in 1994 (Fig. 3f).

The fourth most frequently reported strain had 109 reports ("strain D", spoligotype SB2011, strain ID 121 in Appendix A). Geographic distribution and host species distribution: strain D appeared to have been a localized outbreak, only reported from cattle in Colorado in 2010 (Appendix C, Fig. 1a). Closely related strains: strain D was in another, fourth, spoligotype family. All other strains in the same clonal complex and spoligotype family with strain D were reported from cattle (Fig. 4 and Appendix C, Fig. 1b). The founder of the clonal complex was reported in both US and Mexican cattle, and its single and double locus variants were reported from both the US and Mexico, again making a single country of origin unclear.

The fifth most frequently reported strain (87 reports, "strain E", spoligotype SB0271, strain ID 78 in Appendix A) had the same VNTR profile as the widespread strain $B$, but a different spoligotype. Geographic distribution and host species distribution: strain $\mathrm{E}$ was reported from both wild deer and cattle from 2005 through 2009 in the state of Minnesota. Outside of Minnesota, there was one report of strain E from Nebraska in 2008 (fed steer originating from Minnesota) and three from Texas in 2012, all in cattle (Appendix C). Closely related strains: strain E was in the same clonal complex as strain B (Fig. 3d). Strain E's two single locus variants were reported from wild deer in 2007, also in Minnesota.

These five strains, which account for over half the reports in the database, illustrate the amount of variability in geographic distributions and host affiliations among strains. Their distinct distribution patterns are consistent with several different sources or mechanisms contributing to the maintenance of $M$. bovis in US cattle. 
Strain A

a



b

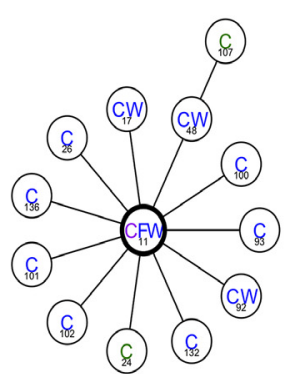

Strain B

C

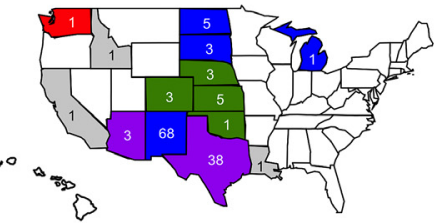

Strain C

e

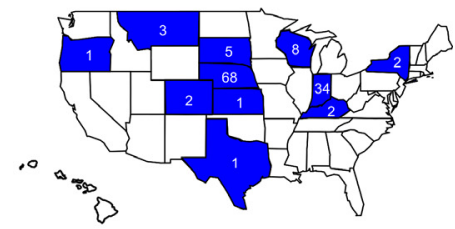

$\mathbf{f}$
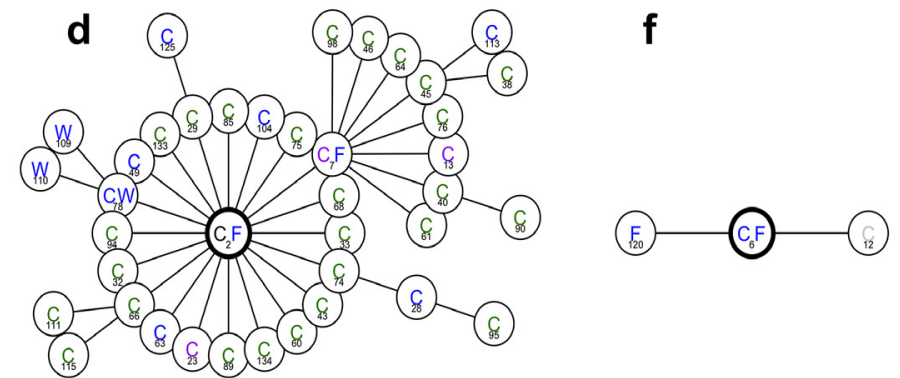

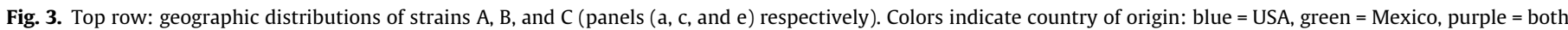

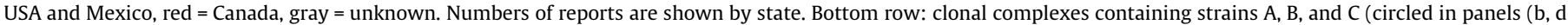

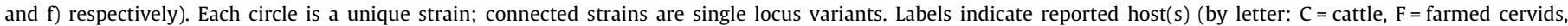

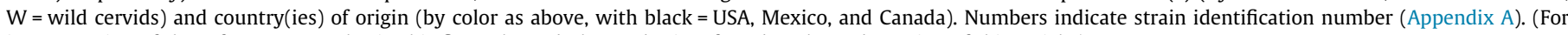
interpretation of the references to color in this figure legend, the reader is referred to the web version of this article.)

US Cattle, $n=555$



Mex Cattle, $n=189$
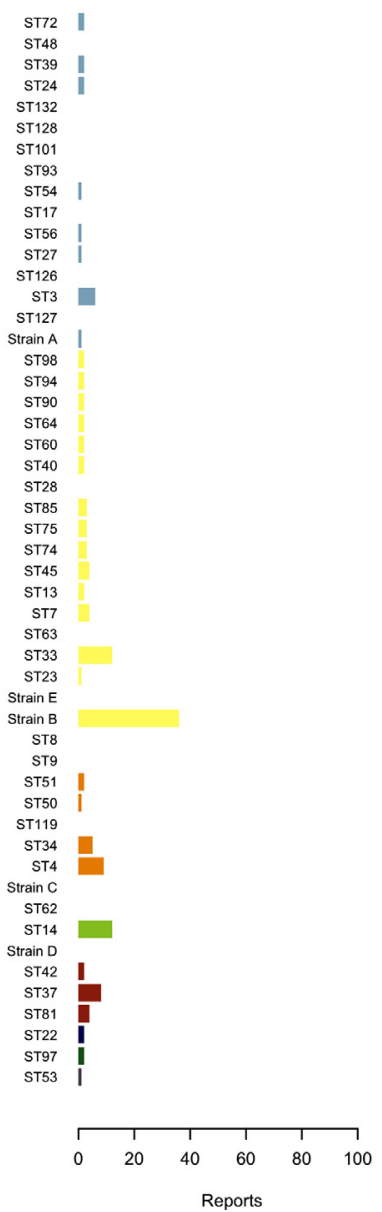

Farmed Cervids, $\mathrm{n}=117$

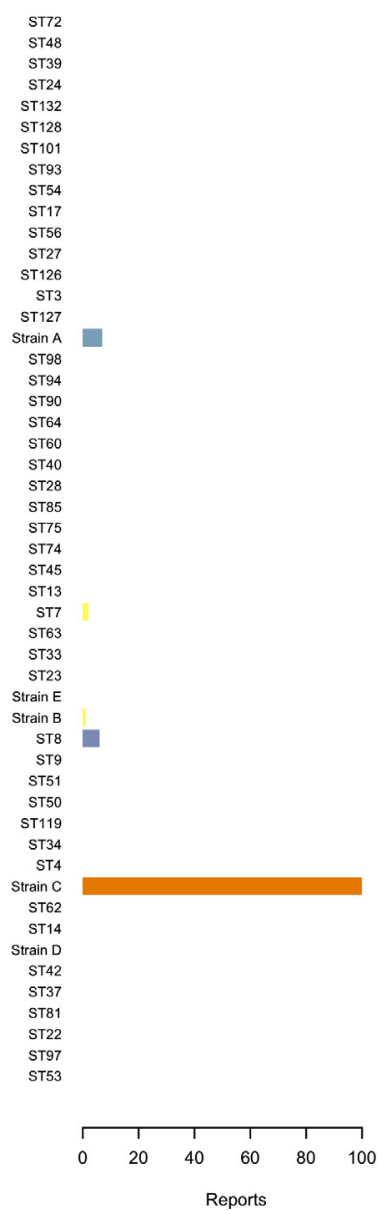

Wild Cervids, $\mathrm{n}=60$




interpretation of the references to colour in this figure legend, the reader is referred to the web version of this article.) 


\subsection{Other strains by host group}

\subsubsection{Wild cervids}

All strains reported from wild cervids fell within the same two spoligotype families and clonal complexes as the high-frequency strains $A$ and $B$, and most of these strains were also reported from cattle (Fig. 4). The close relatedness of these strains is consistent with contact occurring between these hosts and relatively recent transmission events.

\subsubsection{Farmed cervids}

The strain profile of wild cervids strikes a contrast with farmed cervids, which are predominantly infected with a strain genetically distinct from those found in cattle. In addition to strain $C$, a strain in yet another spoligotype family (strain ID 8 in Appendix A) was only reported from farmed cervids (Fig. 4).

\subsubsection{Imported cattle}

Cattle of US and Mexican origin are also infected with closely related strains; in other words, there is little national-scale geographic structure in $M$. bovis genetic relatedness (Fig. 4). The largest clonal complexes, founded by the highest-frequency strains, contain strains found only in the US, only in Mexico, and strains found in both. The highest-frequency strains themselves are found in both the US and Mexico. The years of reports for each strain provide no clear pattern of strains consistently being reported from Mexico prior to appearing in the US, or vice versa.

\section{Discussion}

\subsection{Sampling}

Although our inferences are invariably limited to samples from infected animals reported to the NVSL database, these are the best data available at this spatial and temporal scale, especially considering the low overall prevalence of bovine tuberculosis infection. The rarefaction curve analysis indicates there are more strains in circulation than are included in this dataset, especially among Mexican cattle, but we appear to have captured most of the strains circulating among US cattle. A potential consequence of undersampling is that we may have identified strains as unique to US cattle that are actually present, but undetected, in these other groups. Additional unsampled $M$. bovis diversity in these groups may reveal relationships to other US cattle strains. These possibilities are addressed in the context of our analyses for each group.

\subsection{Variable distribution patterns suggest multiple sources/ mechanisms}

Multiple, strain-dependent drivers likely generated the observed species-geography patterns of $M$. bovis in the U.S., rather than a single external source driving transmission for all of these cases. The dominance of a few high-frequency strains in large clonal complexes, alongside an array of less frequent and more distantly related strains, is consistent with observations in other regions of the world (Gutiérrez Reyes et al., 2012; Smith, 2012; Smith et al., 2003). There also are notable differences among strains in geographic range, with some reported in only a single state while others are widespread, and host range, with one strain associated with farmed cervids and another found in multiple host species. Local and host-specific factors should therefore be considered in plans to control $M$. bovis transmission.

\subsection{Wild cervid strains similar to cattle strains}

Strains reported from wild cervids were either also reported from cattle or were single locus variants of strains in cattle, and most of the other strains in these clonal complexes were from cattle only. Studies focused on outbreaks in Michigan (O'Brien et al., 2006) and Minnesota (Carstensen and DonCarlos, 2011) suggest that wild cervids were initially infected by cattle, as opposed to being the source of infection for cattle, in these cases. However, once infection has been established in wild cervid populations, the degree to which they subsequently impact infection among cattle populations is unknown. Finer resolution genetic data may elucidate the direction of transmission between specific cattle and cervid herds.

With many more cattle sampled than wild cervids, sampling bias is a concern. However, wildlife sampling does tend to be focused on areas with known ongoing $M$. bovis infections (i.e., Michigan), so the wildlife strains with the most potential for transmission to and from US cattle have been sampled and that diversity has been characterized here. Nonetheless, there still may be unsampled wildlife populations carrying other strains infecting US cattle, warranting further wildlife data collection, particularly in areas and host species in regions where cattle have historically been infected.

Because reports from other wildlife species were mostly from Michigan, like most wild cervid reports, we examined them to see if patterns were consistent with our findings. These infections in other wildlife were presumably acquired while scavenging on infected deer carcasses (Berentsen et al., 2011), and thus are likely secondary infections, rather than indicators of primary bTB sources. Indeed, 63 of the 82 reports were of strain A, with 42 from coyotes, 14 from opossums, and 7 from raccoons. Of the remaining 19 reports, all are in the same spoligotype family as strain A, except for one singleton strain in its own spoligotype family (strain ID 99 in Appendix A).

\subsection{Genetically distinct strain associated with farmed cervids}

Although the rarefaction analysis showed similarly low diversity in both farmed and wild cervids, farmed cervids were predominantly infected with strain C (Fig. 4), which was in a genetically distinct spoligotype family from the highest-frequency strains A and $B$. In contrast, wild cervids host a variety of strains either found in cattle or closely related to cattle strains. The relatively strong association between strain $C$ and farmed cervid hosts is consistent with maintained (endemic) transmission among farmed cervids, with occasional spillover to cattle.

The apparent absence of these strains in wild cervids supports our separation of farmed and wild cervids into epidemiologically distinct host groups, despite their taxonomic similarity. Transmission from a cervid game farm to local wild cervids has been reported in the past (Hunter, 1996), but these occurrences seem to be rare. It is possible that unsampled wildlife populations could carry these strains, acting as a link between farmed cervids and cattle. However, given that farmed cervids and wild cervids shared only strain A in this dataset, transmission appears to be infrequent.

Additional sampling among farmed cervids may uncover strains that are more similar to those found in cattle. However, since the dominance of strain $C$ in farmed cervids is already detectable from this sample size, the pattern is unlikely to change with additional data. Furthermore, these reports from farmed cervids are quite widely distributed across the US, so this dominance is not limited to a localized area, as might be expected if a single bTB source was driving the low strain diversity among farmed cervids. 


\subsection{US and Mexican strains similar}

Several patterns of $M$. bovis identity and distribution are similar among US and Mexican cattle. The first and second highest-frequency strains in US cattle match the first (SB0673)- and third (SB0145)-highest frequency spoligotypes in Mexico (Gutiérrez Reyes et al., 2012). These spoligotypes have also been reported from the British Isles (Smith et al., 2003), reflecting a shared history far earlier than the scope of this dataset.

We were not able to identify any strains that had clearly originated from Mexican cattle and subsequently infected US cattle. Such events may have occurred prior to data collection, but this is only a partial explanation, given the steady stream of imports throughout the twenty-year dataset. Another possibility is that VNTR profiles may not be of sufficient resolution to detect strains in the US and Mexico that are actually different, and appear to be the same in these analyses. However, low resolution would only affect our interpretation for those strains reported in both the US and Mexico. Our assumption that infection in all Mexican cattle was acquired in Mexico could also partially explain this lack of structure. Alternatively, some strains may have been acquired after Mexican cattle arrived in the US, misleading our interpretation of geographic structure. However, detection at slaughter is limited to those animals with visible advanced disease, which have likely been infected for some time (USDA: APHIS, 2009). Animals progressing to this stage of disease in non-bTB-endemic regions like the US should be few, and have minimal impact on this analysis.

\subsection{Endemic transmission}

Though we focused on external sources of $M$. bovis infection, we should not overlook the role of endemic transmission among cattle in maintaining and possibly generating additional $M$. bovis diversity. The high-frequency strains A and B have several single locus variants that have only been reported from US cattle, which could be a result of diversification occurring within the US, independently of external introductions. However, the evidence for many unobserved strains in Mexican cattle precludes differentiating whether such variants were generated among U.S. cattle or from unobserved imported strains. Cattle-to-cattle transmission has been shown to contribute substantially to $M$. bovis persistence in Great Britain, even with movement restrictions and test-andslaughter protocols in place (Conlan et al., 2012). M. bovis is transmitted directly among US cattle, as is evident from epidemiologic traceback investigations (USDA: APHIS, 2011), but to what degree this transmission route contributes to maintaining overall $M$. bovis diversity is unknown.

\subsection{Conclusions and future directions}

The external sources we examined here appear to play different roles with respect to infecting US cattle with $M$. bovis. Of the three groups analyzed, only farmed cervids showed a pattern of endemic maintenance of genetically distinct strains, with occasional spillover to cattle. Conversely, wild cervids appear to acquire most of their strains from cattle, but may then maintain and possibly amplify transmission of those strains among themselves and cattle. Infected Mexican cattle are regularly reported, but the degree to which they subsequently infect US cattle is unclear, due to the genetically similar backgrounds of bTB in these host groups.

Whole genome sequencing data could potentially resolve many of the ambiguities encountered here. Where numerous individual cattle (and cervids) are infected with the same VNTR-defined strain, improved resolution could identify herd-to-herd level transmission events (Biek et al., 2012). This approach could clarify the directionality of transmission between external sources and US cattle. Furthermore, identifying structure within the high-frequency strains would allow us to ask more detailed questions about $M$. bovis transmission within the US, such as how livestock shipment patterns affect pathogen spread. A better understanding of the mechanisms driving $M$. bovis maintenance in US cattle can ultimately inform targeted control measures to reduce infection and disease.

\section{Acknowledgements}

Funding provided by USDA Cooperative Agreement 11-92080296-CA 11-1. CTW acknowledges the Research and Policy for Infectious Disease Dynamics (RAPIDD) Program, Science and Technology Directorate, US Department of Homeland Security, and Fogarty International Center, National Institutes of Health; Foreign Animal Disease Modeling Program, Science and Technology Directorate, U. S. Department of Homeland Security (Grant ST-108000017). However, the views and conclusions contained in this document are those of the authors and should not be interpreted as necessarily representing the official policies, either expressed or implied, of USDA-APHIS-Veterinary Services or the US Department of Homeland Security. We also acknowledge the National Institute for Mathematical and Biological Synthesis for supporting the Modeling Bovine Tuberculosis working group. Comments from two anonymous reviewers greatly improved the manuscript.

\section{Appendix A. Supplementary data}

Supplementary data associated with this article can be found, in the online version, at http://dx.doi.org/10.1016/j.meegid.2014. 09.025 .

\section{References}

Allen, A., Dale, J., McCormick, C., Mallon, T., Costello, E., Gordon, S., Hewinson, R. Skuce, R., Smith, N., 2013. The phylogeny and population structure of Mycobacterium bovis in the British Isles. Infect. Genet. Evol. 20, 8-15.

Bengis, R., Kriek, N., Keet, D., Raath, J., De Vos, V., Huchzermeyer, H., 1996. An outbreak of bovine tuberculosis in a free-living African buffalo (Syncerus caffersparrman) population in the Kruger National Park: a preliminary report. Onderstepoort J. Vet. Res. 63, 15.

Berentsen, A.R., Dunbar, M.R., Johnson, S.R., Robbe-Austerman, S., Martinez, L., Jones, R.L., 2011. Active use of coyotes (Canis latrans) to detect Bovine Tuberculosis in northeastern Michigan, USA. Vet. Microbiol. 151, 126-132.

Biek, R., O'Hare, A., Wright, D., Mallon, T., McCormick, C., Orton, R.J., McDowell, S. Trewby, H., Skuce, R.A., Kao, R.R., 2012. Whole genome sequencing reveals local transmission patterns of Mycobacterium bovis in sympatric cattle and badger populations. PLoS Pathog. 8, e1003008.

Carstensen, M., DonCarlos, M.W., 2011. Preventing the establishment of a wildlife disease reservoir: a case study of bovine tuberculosis in wild deer in Minnesota, USA. Vet. Med. Int. 2011.

Collins, D., Gabric, D., De Lisle, G., 1988. Typing of Mycobacterium bovis isolates from cattle and other animals in the same locality. N. Z. Vet. J. 36, 45-46.

Conlan, A.J.K., McKinley, T.J., Karolemeas, K., Pollock, E.B., Goodchild, A.V., Mitchell, A.P., Birch, C.P.D., Clifton-Hadley, R.S., Wood, J.L.N., 2012. Estimating the hidden burden of bovine tuberculosis in great Britain. PLOS Comput. Biol. 8, e1002730.

Feil, E.J., Li, B.C., Aanensen, D.M., Hanage, W.P., Spratt, B.G., 2004. EBURST: inferring patterns of evolutionary descent among clusters of related bacterial genotypes from multilocus sequence typing data. J. Bacteriol. 186, 1518-1530.

Gutiérrez Reyes, J.A., Casanova, L.G., Torres, C.R., Gallegos, S.L.S., Alarcón, G.J.C. Pezzat, M.M., Martínez, O.P., Chávez, C.E., Suazo, F.M., 2012. Population structure of Mycobacterium bovis isolates from cattle in Mexico. Prev. Vet. Med. 106, 1-8.

Hunter, D.L., 1996. Tuberculosis in free-ranging, semi free-ranging and captive cervids. Rev. Sci. Tech. 15, 171-181.

Martinez, L.R., Harris, B., Black, W.C., Meyer, R.M., Brennan, P.J., Vissa, V.D., Jones, R.L., 2008. Genotyping North American animal Mycobacterium bovis isolates using multilocus variable number tandem repeat analysis. J. Vet. Diagn. Invest. 20, 707-715.

Miller, R.S., Sweeney, S.J., 2013. Mycobacterium bovis (bovine tuberculosis) infection in North American wildlife: current status and opportunities for mitigation of risks of further infection in wildlife populations. Epidemiol. Infect. 141, 13571370 . 
Naranjo, V., Gortazar, C., Vicente, J., de la Fuente, J., 2008. Evidence of the role of European wild boar as a reservoir of Mycobacterium tuberculosis complex. Vet. Microbiol. 127, 1-9.

O’Brien, D.J., Schmitt, S.M., Fitzgerald, S.D., Berry, D.E., Hickling, G.J., 2006. Managing the wildlife reservoir of Mycobacterium bovis: the Michigan, USA, experience. Vet. Microbiol. 112, 313-323.

Oksanen, J., Guillaume Blanchet, F., Kindt, R., Legendre, P., Minchin, P.R., O'Hara, R.B. Simpson, G.L., Solymos, P., Stevens, M.H.H., Wagner, H., 2012. vegan Community Ecology Package, $\mathrm{R}$ package version $2.0-5 .<$ http://CRAN.Rproject.org/package=vegan>

R Core Team, 2012. R: A Language and Environment for Statistical Computing. R Foundation for Statistical Computing.

Reyes, J.F., Francis, A.R., Tanaka, M.M., 2008. Models of deletion for visualizing bacterial variation: an application to tuberculosis spoligotypes. BMC Bioinformatics 9, 496.

Smith, N.H., 2012. The global distribution and phylogeography of Mycobacterium bovis clonal complexes. Infect. Genet. Evol. 12, 857-865.

Smith, N.H., Berg, S., Dale, J., Allen, A., Rodriguez, S., Romero, B., Matos, F., Ghebremichael, S., Karoui, C., Donati, C., 2011. European 1: a globally important clonal complex of Mycobacterium bovis. Infect. Genet. Evol. 11, 1340-1351.

Smith, N.H., Dale, J., Inwald, J., Palmer, S., Gordon, S.V., Hewinson, R.G., Smith, J.M., 2003. The population structure of Mycobacterium bovis in Great Britain: clonal expansion. Proc. Natl. Acad. Sci. 100, 15271-15275.
Smith, N.H., Gordon, S.V., de la Rua-Domenech, R., Clifton-Hadley, R.S., Hewinson, R.G., 2006. Bottlenecks and broomsticks: the molecular evolution of Mycobacterium bovis. Nat. Rev. Microbiol. 4, 670-681.

Smith, N.H., Upton, P., 2012. Naming spoligotype patterns for the RD9-deleted lineage of the Mycobacterium tuberculosis complex. Infect. Genet. Evol. 12, 873876 http://www.Mbovis.org.

Smith, P., 1968. Bovine-type tuberculosis infection in feral swine. Vet. Med., University of California, Davis, Davis, California, p. 28.

USDA: APHIS, 2009. Analysis of Bovine Tuberculosis Surveillance in Accredited Free States, <http://www.aphis.usda.gov/vs/nahss/cattle/tb_2009_evaluation_of_tb_ in_accredited_free_states_jan_09.pdf> (Accessed May 13 2014).

USDA: APHIS, 2011. Assessment of Pathways for the Introduction and Spread of Mycobacterium bovis in the United States, 2009, <http://www.aphis.usda.gov/ animal_health/emergingissues/downloads/bovine_tb_pathways_2009030711.pdf> (Accessed September 3 2014).

USDA: APHIS, 2013. United States - Mexico Joint Strategic Plan for Collaboration on Bovine Tuberculosis, <http://www.aphis.usda.gov/animal_health/downloads/ US-MX_TB_Strategic_Plan_Eng_2013.pdf> (Accessed September 9 2014).

Wobeser, G., 2009. Bovine tuberculosis in Canadian wildlife: an updated history. Can. Vet. J. 50, 1169

Woodroffe, R., Donnelly, C., Johnston, W., Bourne, F., Cheeseman, C., Clifton-Hadley, R., Cox, D., Gettinby, G., Hewinson, R., Le Fevre, A., 2005. Spatial association of Mycobacterium bovis infection in cattle and badgers Meles meles. J. Appl. Ecol. 42, 852-862. 